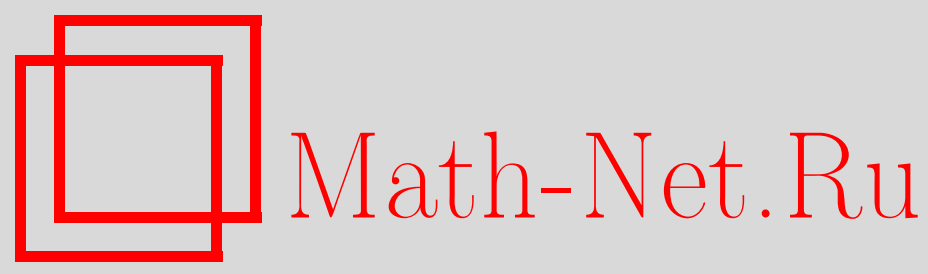

И. Д. Тверитинов, Связь между различными реализациями преобразований Боголюбова, Матем. заметки, 2004, том 75, выпуск 5, 797-800

DOI: https://doi.org/10.4213/mzm560

Использование Общероссийского математического портала Math-Net.Ru подразумевает, что вы прочитали и согласны с пользовательским соглашением http://www . mathnet.ru/rus/agreement

Параметры загрузки:

IP: 35.173 .219 .12

26 апреля 2023 г., $17: 23: 04$

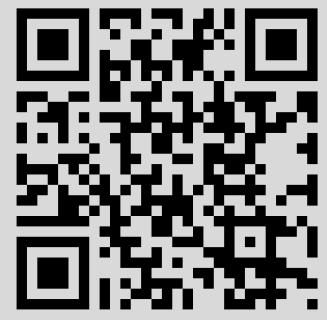




\section{СВЯЗЬ МЕЖДУ РАЗЛИЧНЫМИ РЕАЛИЗАЦИЯМИ ПРЕОБРАЗОВАНИЙ БОГОЛЮБОВА}

\section{И. Д. Тверитинов}

В заметке пойдет речь о различных подходах, испольуемых при реализациях автоморфизмов алгебры Гейзенберга (представляющей канонические коммутационные соотношения) как алгебры операторов (вообще говоря, неограниченных) в гильбертовом пространстве. В работе [1] рассматриваются линейные преобразования (называемые там каноническими) операторов алгебры Гейзенберга, реализованной как алгебра операторов в пространстве Баргмана-Фока, и показано, что некоторые (там они называются собственными) из этих линейных преобразований (преобразований Боголюбова) могут реализовываться с помощью действия сопряжением некоторой подгруппы группы унитарных операторов пространства Баргмана-Фока. Для каждого собственного преобразования в [1] найдены явные формулы для унитарного оператора в пространстве Баргмана-Фока (унитарного преобразования Боголюбова), действие которого реализует соответствующее собственное преобразование. В работах [2]-[4], рассматриваются линейные преобразования операторов алгебры Гейзенберга (преобразования Боголюбова), реализованной операторами в пространстве Винера-Сигала-Фока, порожденные симплектическими преобразованиями комплексного гильбертова пространства (с естественной симплектической структурой), по которому построена алгебра Гейзенберга (посредством центрального расширения при помощи алгебры $\mathbf{R}$; подробнее см. [3]). В предлагаемой заметке устанавливается связь между преобразованиями Боголюбова, действующими в различных реализациях алгебры Гейзенберга, и из явных формул для унитарных преобразований Боголюбова выводятся свойства отображения, осуществляющего эквивалентность рассматриваемых представлений алгебры Гейзенберга.

1. Предварительные сведения. Всюду далее термины оператор, преобразование и отображение считаются синонимами. Пусть $H$ - комплексное гильбертово пространство, полученное путем комплексификации вещественного гильбертова пространства $Q$; таким образом, вещественное гильбертово пространство $H$ совпадает с (=изоморфно) $Q \oplus i P$, где $P$ - вещественное гильбертово пространство, являющееся копией пространства $Q$. Пространством Винера-Сигала-Фока назьвается пополнение $L_{2}\left(Q, \mu_{1 / 2}\right)$ пространства (непрерывных) цилиндрических функций на $Q$, квадратично интегрируемых относительно гауссовской меры $\mu_{1 / 2}$ на $Q$, корреляцинный оператор которой равен половине единичного оператора; при этом существует естественный изоморфизм пространства $L_{2}\left(Q, \mu_{1 / 2}\right)$ на пространство $L_{2}\left(Q_{-}, \mu_{1 / 2}\right)$, где символом $Q_{-}$обозначено расширение пространства $Q$, на алгебре цилиндрических подмножеств которого образ гауссовской меры $\mu_{1 / 2}$ (обозначаемьй тем же символом) представляет собой счетно аддитивную меру [5] (образ каждой функции из $L_{2}\left(Q, \mu_{1 / 2}\right)$ при этом изоморфизме можно считать ее продолжением на $\left.Q_{-}\right)$.

Пространство $F(H)=\bigoplus_{n=0}^{\infty}\left(\otimes^{n} H\right)$ с гильбертовой нормой $\|\cdot\|_{F(H)}=\sum_{n=0}^{\infty}\|\cdot\|_{H^{n}, \text { где }}$ $H^{n}=H \otimes \cdots \otimes H$, называется пространством Фока. Образ оператора симметритизации

$P \vee: F(H) \ni f_{1} \otimes \cdots \otimes f_{n} \mapsto f_{1} \vee \cdots \vee f_{n}, \quad$ где $f_{1} \vee \cdots \vee f_{n}=\frac{1}{\sqrt{n !}} \sum_{\sigma \in S_{n}} f_{\sigma(1)} \otimes \cdots \otimes f_{\sigma(n)}$,

назьвается симметрическим пространством Фока и обозначается через $F \vee(H)$. Обозначим еще через $L_{2}\left(H, \mu_{1 / 2}\right)$ гильбертово пространство, полученное путем пополнения пространства цилиндрических аналитических функций на $H$, квадрат которых интегрируем по гауссовской цилиндрической мере $\mu_{1 / 2}$, определенной на алгебре цилиндрических множеств пространства $H$ (при этом функция $f$ назьвается иилиндрической, если $f(h)=f(P(h)), h \in H, P$ - конечномерный комплексно-линейньй проектор). Пространство $L_{2}\left(H, \mu_{1 / 2}\right)$ называется пространством Барحмана-Фока.

Работа выполнена при поддержке Российского фонда фундаментальных исследований, грант № 02-01-01074. 
Операторы $\hat{q}_{a}$ и $\hat{p}_{a}$ в пространстве Винера-Сигала-Фока (ВСФ), определяемые равенствами

$$
\begin{aligned}
& \hat{q}_{a}: L_{2}\left(Q, \mu_{1 / 2}\right) \ni f \mapsto[x \mapsto\langle x, a\rangle f(x)], \\
& \hat{p}_{a}: L_{2}\left(Q, \mu_{1 / 2}\right) \ni f \mapsto[x \mapsto-\operatorname{idf}(x)(a)+i\langle x, a\rangle f(x)],
\end{aligned}
$$

являются самосопряженными в существенном на области, представляющей собой алгебраическое тензорное произведение счетного числа копий пространств Шварца (предполагается, что в пространстве $Q$ выбран ортонормированньй базис и что функции из соответствующих пространств Шварца зависят только от переменных, отвечающих определенному базисному вектору пространства $Q)$, назьваются операторами представления Шрёдингера алгебры Гейзенберга. Иногда удобнее рассматривать операторы

$$
a_{v s}(x, y)=\frac{\hat{q}_{x}-i \hat{q}_{y}+\hat{p}_{y}+i \hat{p}_{x}}{\sqrt{2}}, \quad a_{v s}^{*}(x, y)=\frac{\hat{q}_{x}+i \hat{q}_{y}+\hat{p}_{y}-i \hat{p}_{x}}{\sqrt{2}} .
$$

В симметрическом пространстве Фока и в пространстве Баргмана-Фока операторы

$$
\begin{aligned}
& a_{F}^{*}(f): F_{\vee}(H) \ni f_{1} \vee \cdots \vee f_{n} \mapsto f \vee f_{1} \vee \cdots \vee f_{n} \\
& a_{F}(f): F_{\vee}(H) \ni f_{1} \vee \cdots \vee f_{n} \mapsto \sum_{i=1}^{n}\left\langle f, f_{i}\right\rangle \cdot f_{1} \vee \cdots \vee f_{i-1} \vee f \vee f_{i+1} \vee \cdots \vee f_{n}
\end{aligned}
$$

и, соответственно, операторы

$$
\begin{aligned}
& \hat{a}_{b}(f): L_{2}\left(H, \mu_{1 / 2}\right) \ni G \mapsto\left[H \ni h \mapsto \frac{d G(h)}{d h}(f)\right], \\
& \hat{a}_{b}^{*}(f): L_{2}\left(H, \mu_{1 / 2}\right) \ni G \mapsto[H \ni h \mapsto\langle h, f\rangle G(h)]
\end{aligned}
$$

играют ту же роль, что и операторы $a_{v s}(x, y), a_{v s}^{*}(x, y)$, действующие в пространстве Винера-Сигала-Фока (см. [6], [1]): для этих семейств операторов выполняются канонические коммутационные соотношения (KKC):

$$
[\hat{a}(f), \hat{a}(g)]=\left[\hat{a}^{*}(f), \hat{a}^{*}(g)\right]=0, \quad\left[\hat{a}(f), \hat{a}^{*}(g)\right]=\langle f, g\rangle_{C} I
$$

(имеется ввиду, что семейство операторов $\{\hat{a}(f), \hat{a}(g)\}$ - одно из семейств $\left\{\hat{a}_{v s}(x, y), \hat{a}_{v s}^{*}(x, y)\right\}$, $\left.\left\{\hat{a}_{F}(f), \hat{a}_{F}^{*}(f)\right\},\left\{\hat{a}_{b}(f), \hat{a}_{b}^{*}(f)\right\}\right)$. Предполагается, что все операторы имеют общую плотную область определения и звездочка означает сопряжение относительно двойственности, порожденной эрмитовым произведением. Говорят, что представление $\mathrm{KKC}$ операторами $\left\{\hat{a}(f), \hat{a}^{*}(f)\right\}$ в гильбертовом пространстве обладает вакуумным вектором $v_{0}$, если для всех $f \in H$ справедливо равенство $\hat{a}(f)\left(v_{0}\right)=0$, причем множество векторов вида $\hat{a}^{*}\left(f_{1}\right) \cdot \hat{a}^{*}\left(f_{2}\right) \cdots \hat{a}^{*}\left(f_{n}\right)\left(v_{0}\right)$ тотально. Для всякого такого представления $\mathrm{KKC}$, по-прежнему будут использоваться обозначения $\hat{q}_{a}, \hat{p}_{a}$, $a \in Q=P$, для операторов, определяемых соотношениями

$$
\hat{a}(x, y)=\frac{\hat{q}_{x}-i \hat{q}_{y}+\hat{p}_{y}+i \hat{p}_{x}}{\sqrt{2}}, \quad \hat{a}^{*}(x, y)=\frac{\hat{q}_{x}+i \hat{q}_{y}+\hat{p}_{y}-i \hat{p}_{x}}{\sqrt{2}},
$$

где $f=(x, y) \in H=Q \oplus P$.

Как известно (см., например, книгу [1]) все представления $\mathrm{KKC}$, обладающие вакуумньмм вектором, унитарно эквивалентны.

2. Преобразования операторов, сохраняющие ККС. Отметим, что всякий оператор в пространстве $H=Q \oplus P$ задается набором операторов $(\alpha, \beta, \gamma, \delta)$, где $\alpha, \beta, \gamma, \delta$ - операторы, действующие соответственно из $Q$ в $Q$, из $P$ в $Q$, из $Q$ в $P$, из $P$ в $P$. Для всякого представления $\mathrm{KKC}$ операторами $\left\{\hat{a}(f), \hat{a}^{*}(f)\right\}$, действующими в некотором гильбертовом пространстве, всякий оператор в пространстве $H$ индуцирует оператор в алгебре операторов, порожденной операторами $\left\{\hat{a}(f), \hat{a}^{*}(f)\right\}$ (или, что то же самое, в алгебре, порожденной операторами $\left\{\hat{q}_{a}, \hat{p}_{a}\right\}$, являющимися 
элементами той же алгебры). Для каждого линейного отображения $A: H \rightarrow H$ оператор, действующий в алгебре операторов, порожденной операторами представления $\mathrm{KKC}$, обозначается через $\Pi_{A}$ и определяется так: $\Pi_{A}: \hat{q}_{a} \mapsto \hat{q}_{\alpha^{*}(a)}+\hat{p}_{\beta^{*}(a)}, \Pi_{A}: \hat{p}_{a} \mapsto \hat{q}_{\gamma^{*}(a)}+\hat{p}_{\delta^{*}(a)}$. Условия того, что оператор $\Pi_{A}$ сохраняет $\mathrm{KKC} \mathrm{имеют} \mathrm{вид:} \delta^{*} \alpha-\beta^{*} \gamma=\mathrm{id}, \delta^{*} \beta=\beta^{*} \delta, \gamma^{*} \alpha=\alpha^{*} \gamma$. Группа всех обратимых вещественно линейных преобразований $A$ пространства $H$ таких, что соответствующие преобразования алгебры операторов представления $\mathrm{KKC} \Pi_{A}$ сохраняют $\mathrm{KKC}$, назьвается симплектической әруппой.

Если $\left\{\hat{a}(f), \hat{a}^{*}(f)\right\}$ - представление ККС операторами в гильбертовом пространстве $E$, обладающее вакуумным вектором и для симплектического преобразования $A$ существует унитарное преобразование Боголюбова $V_{A}$ такое, что для всякого $a \in Q$ выполняются равенства $V_{A}^{-1} \cdot \hat{q}_{a} \cdot V_{A}=$ $\Pi_{A}\left(\hat{q}_{a}\right), V_{A}^{-1} \cdot \hat{p}_{a} \cdot V_{A}=\Pi_{A}\left(\hat{p}_{a}\right)$, то будем говорить, что автоморфизм $\Pi_{A}$ приводим и симплектическое преобразование порождает унитарное преобразование Боголюбова. Согласно теореме Шейла [7] симплектическое преобразование $A$ приводимо тогда и только тогда, когда оператор $\sqrt{A^{*} \cdot A}-I \in L_{2}(H)$, где через $L_{2}(H)$ обозначается пространство операторов Гильберта-Шмидта. Если симплектическое преобразование $A$ приводимо, то унитарный оператор $V_{A}$ определяется с точностью до комплексного множителя, равного по модулю единице, т.е. многозначное отображение $A \mapsto V_{A}$ индуцирует проективное представление подгруппы симплектической группы, состоящее из всех приводимых симплектических преобразований, операторами, действующими в проективном пространстве, построенном по гильбертовому пространству, в котором действуют операторы представления KKC. В случае, когда пространство представления КKC реализовано как пространство Винера-Сигала-Фока, существуют явные формулы для унитарных преобразований Боголюбова (см. [3], [4] и имеющиеся там ссылки).

В книге [1] рассматриваются преобразования алгебры операторов представления KKC (реализованной при помощи операторов в пространстве Фока), аналогичные преобразованиям $\Pi_{A}$; в этой книге они называются линейными каноническими преобразованиями. Линейное каноническое преобразование определяется при помощи пары $\Phi, \Psi$ комплексно-линейных преобразований пространства $H$; при этом соответствующее преобразование алгебры операторов представления KKC, обозначаемое через $\Gamma_{\Phi, \Psi}$, задается так: $\Gamma_{\Phi, \Psi}: a_{F}(f) \mapsto a_{F}\left(\Phi^{\prime}(f)\right)+a_{F}^{*}\left(\Psi^{*}(\bar{f})\right)$, где $\bar{f}$ обозначает сопряжение, определяемое выбором подпространства $Q$ в качестве действительной части пространства $H$, а через $\Phi^{\prime}$ обозначен оператор, определяемьй равенством $\Phi^{\prime}(f)=\bar{\Phi}^{*}(\bar{f})$. Для того чтобы преобразование $\Gamma_{\Phi, \Psi}$ сохраняло $\mathrm{KKC}$, необходима и достаточна справедливость равенств $\Phi \cdot \Psi^{\prime}-\Psi \cdot \Phi^{\prime}=0, \Phi \dot{\Phi}^{*}-\Psi \cdot \Psi^{*}=I, \bar{\Phi} \dot{\Psi}^{*}-\bar{\Psi} \cdot \Phi^{*}=0, \bar{\Phi} \cdot \Phi^{\prime}-\bar{\Psi} \cdot \Psi^{\prime}=I$. Вопрос о связи между преобразованиями $\Pi_{A}$ и $\Gamma_{\Phi, \Psi}$ решает следующая теорема.

Tеорема 1. Пусть $\left\{\hat{a}(f), \hat{a}^{*}(f)\right\}$ - представление $\mathrm{KKC,} \mathrm{операторами} \mathrm{в} \mathrm{некотором}$ зильбертовом пространстве, обладающее вакуумным вектором. Предполохим, что преобразования $\Phi, \Psi$ задают автоморфизм алгебры операторов $\mathrm{KKC} \mathrm{(обозначаемый} \mathrm{че-}$ рез $\left.\Gamma_{\Phi, \Psi}\right)$. Тогда преобразование $A: H=Q \oplus P \rightarrow H=Q \oplus P$, определяемое матрицей

$$
\left(\begin{array}{cc}
\operatorname{Re}(\Phi+\Psi) & \operatorname{Im}(\Phi-\Psi) \\
-\operatorname{Im}(\Phi+\Psi) & \operatorname{Re}(\Phi-\Psi)
\end{array}\right)
$$

симплектическое, и определяемый им автоморфизм $\Pi_{A}$ совпадает с автоморфизмом $\Gamma_{\Phi, \Psi}$

По аналогии с $V_{A}$ определим $W_{\Phi, \Psi}$ как унитарный оператор такой, что $\forall f \in H$ верно равенство $W_{\Phi, \Psi}^{-1} \cdot a(f) \cdot W_{\Phi, \Psi}=\Gamma_{\Phi, \Psi}(a(f))$ (существование $W_{\Phi, \Psi}$ не утверждается). Пусть даны два стандартных представления ККС операторами, действующими в пространствах Винера-Сигала-Фока и Фока соответственно. Пусть для операторов $\Phi, \Psi$ существует $W_{\Phi, \Psi}$ - оператор, действующий в пространстве Фока и построенный при помощи стандартного представления КKC операторами, действующими в пространстве Фока. Пусть для симплектического преобразования

$$
A=\left(\begin{array}{cc}
\operatorname{Re}(\Phi+\Psi) & \operatorname{Im}(\Phi-\Psi) \\
-\operatorname{Im}(\Phi+\Psi) & \operatorname{Re}(\Phi-\Psi)
\end{array}\right)
$$

оператор $V_{A}$ построен при помощи представления $\mathrm{KКC} \mathrm{операторами,} \mathrm{действующими} \mathrm{в} \mathrm{пространст-}$ ве Винера-Сигала-Фока. Так как всякие представления КKC, обладающие вакуумным вектором, 
унитарно эквивалентны, из предыдущей теоремы следует, что существует единственный унитарньй оператор, обозначаемьй через $T_{V \rightarrow F}$, действующий из пространства Винера-Сигала-Фока в пространства Фока и переводящий вакуумный вектор в вакуумный, такой, что при некотором выборе операторов $V_{A}, W_{\Phi, \Psi}$ (которые определены с точностью до комплексного множителя равного по модулю единице), верно равенство $T_{V \rightarrow F}^{-1} \cdot W_{\Phi, \Psi} \cdot T_{V \rightarrow F}=V_{A}$. Исползуя некоторые свойства операторов $V_{A}, W_{\Phi, \Psi}$, можно описать действия оператора $T_{V \rightarrow F}$, не выписывая соответствующей явной формулы; именно, справедлива следующая теорема.

Tеорема 2. Пусть $\left\{e_{n}\right\}, n \in \mathbb{N}$, - ортонормированный базис пространства $Q$. Обозначим через $H_{n}(x), n \in \mathbb{N} \cup\{0\}$, последовательность нормированных полиномов Эрмита (рассматриваемых как әлементы пространства $L_{2}\left(\mathbb{R}, \mu_{1 / 2}\right)$ - пространства измеримых квадратично интегрируемых функций по гауссовской мере с половинным корреляиионным оператором), через $S_{n}(z), n \in \mathbb{N} \cup\{0\}$, нормированный полином $z^{n}$ (полином рассматривается как элемент пространства $L_{2}\left(\mathbb{C}, \mu_{1 / 2}\right)$ - измеримых функиий комплексного переменного, квадратично интегрируемых по гауссовской мере с половинным коррелячионным оператором). Тогда множества

$$
\begin{aligned}
& \left\{\prod_{l=1}^{k} H_{n_{l}}\left(\left\langle e_{m_{l}}, x\right\rangle_{\mathbb{R}}\right) \mid n_{i} \in \mathbb{N} \cup\{0\}, m_{i} \in \mathbb{N}, i \neq j \Rightarrow m_{i} \neq m_{j}, n_{i} \neq n_{j}\right\}, \\
& \left\{\prod_{l=1}^{k} S_{n_{l}}\left(\left\langle e_{m_{l}}, z\right\rangle_{\mathbb{C}}\right) \mid n_{i} \in \mathbb{N} \cup\{0\}, m_{i} \in \mathbb{N}, i \neq j \Rightarrow m_{i} \neq m_{j}, n_{i} \neq n_{j}\right\},
\end{aligned}
$$

образуют ортонормированнье базисы в пространствах $L_{2}\left(Q, \mu_{1 / 2}\right), L_{2}\left(H, \mu_{1 / 2}\right)$ соответственно и оператор $T_{V \rightarrow F}$ переводит базисный вектор пространства $L_{2}\left(Q, \mu_{1 / 2}\right)$ в базисный вектор с теми жее индексами в пространстве $L_{2}\left(H, \mu_{1 / 2}\right)$.

\section{СПИСОК ЦИТИРОВАННОЙ ЛИТЕРАТУРЫ}

1. Березин Ф.А. Метод вторичного квантования. М.: Наука, 1965. 2. Купш И., Смолянов О. Г. // Матем. заметки. 2000. Т. 68. №3. С. 474-479. 3. Купш И., Смолянов О. Г. // Докл. РАН. 2000. Т. 371. №1. С. 21-25. 4. Тверитинов И. Д. // Вестн. МГУ. Сер. 1. Матем., мех. 2002. № 4. С. 9-14. 5. Аккарди Л., Смолянов О. Г. // Матем. заметки. 1998. Т. 64. № 4. C. 483-492. 6. Ottesen J. T. Infinite dimensional Groups and Algebras in Quantum Physics. Berlin: Springer, 1995. 7. Лион ЖК., Вернь М. Представление Вейля, индекс Маслова и тэта-ряды. М.: Мир, 1983. 